\title{
On the Existence of Global Weak Solutions to 1D Pollutant Transport Model
}

\author{
Brahima ROAMBA ${ }^{1,2}$, Jean de Dieu ZABSONRE ${ }^{1} \&$ Yacouba $_{\text {ZONGO }}^{2}$ \\ ${ }^{1}$ Institut Universitaire de Technologie, Université Nazi Boni, 01 BP 1091 Bobo-Dioulasso, Burkina Faso \\ ${ }^{2}$ Unité de Formation et de Recherche en Sciences et Techniques, Université Nazi Boni, 01 BP 1091 Bobo-Dioulasso, \\ Burkina Faso
}

Correspondence: Jean de Dieu ZABSONRE, Institut Universitaire de Technologie, Université Nazi Boni, 01 BP 1091 Bobo-Dioulasso, Burkina Faso. E-mail: jzabsonre@gmail.com

Received: May 2, 2017 Accepted: June 13, 2017 Online Published: July 24, 2017

doi:10.5539/jmr.v9n4p124 URL: https://doi.org/10.5539/jmr.v9n4p124

\begin{abstract}
We consider a one-dimensionnal bilayer model coupling shallow water and Reynolds lubrication equations with a molecular interactions between molecules. These molecular interactions give rise to intermolecular forces, namely the long-range van der Waals forces and short-range Born intermolecular forces. In this paper, an expression will be used to take into account all these intermolecular forces. Our model is a similar model studied in (Roamba, Zabsonré\& Zongo, 2017). The model considered is represented by the two superposed immiscible fluids. A similar model was studied in (Zabsonré Lucas \& Fernandez-Nieto, 2009) but the authors do not take into account the intermolecular forces. Without hypothesis about the unknowns as in (Roamba, Zabsonré\& Zongo, 2017), we show the existence of global weak solution in time in a periodic domain.
\end{abstract}

Keywords: shallow water equations, bilayer models, viscosity, friction, capillarity, intermolecular forces

\section{Introduction}

The purpose of this paper is devoted to the existence of global weak solutions to 1D pollutant transport model. This model can be used to simulate for instance the evolution of a pollutant fluid over water. It is a bilayer model of two immiscible fluids where the upper layer can be represented by a Reynolds lubrifications model and the lower layer by a shallow water model.

This work follows the work done in (Roamba, Zabsonré \& Zongo, 2017). In (Roamba, Zabsonré \& Zongo, 2017) as in this present work, we use a model of transport of pollutant in 1D formally derived in (Fernandez-Nieto, Narbona-Reina \& Zabsonré 2013). In (Roamba, Zabsonré \& Zongo, 2017), the authors showed the existence of global weak solutions of similar model derived in (Fernandez-Nieto, Narbona-Reina \& Zabsonré 2013). To lead well this result, the authors considered the condition according to which $h_{2} \leq h_{1}$ (the water layer is more important than the layer of the pollutant). We suppose in this paper the existence of molecular interactions between molecules and this leads us to use the force of Van Der Waals which is given by $V\left(h_{1}\right)=\frac{1}{h_{1}^{3}}-\frac{\alpha}{h_{1}^{4}} \quad(\alpha>0)$, see (Kitavtsev, Laurençot \& Niethammer, 2011; Seemann, Herminghaus \& Jacobs, 2001). This force of Van Der Waals allows us to lower the height of water which allows us to get around hypothesis made in (Roamba, Zabsonré\& Zongo, 2017). The model studied is read as follow:

$$
\begin{gathered}
\partial_{t} h_{1}+\partial_{x}\left(h_{1} u_{1}\right)=0, \\
\partial_{t}\left(h_{1} u_{1}\right)+\partial_{x}\left(h_{1} u_{1}^{2}\right)+\frac{1}{2} g \partial_{x} h_{1}^{2}-4 v_{1} \partial_{x}\left(h_{1} \partial_{x} u_{1}\right)+\frac{u_{1}}{\beta}-h_{1} \partial_{x}\left(\sigma \partial_{x}^{2} h_{1}-V\left(h_{1}\right)\right) \\
r g h_{1} \partial_{x} h_{2}+r g h_{2} \partial_{x}\left(h_{1}+h_{2}\right)+r_{1} h_{1}\left|u_{1}\right|^{2} u_{1}=0 . \\
\partial_{t} h_{2}+\partial_{x}\left(h_{2} u_{1}\right)-\varepsilon \partial_{x}^{2} h_{2}-\partial_{x}\left(\left(a h_{2}{ }^{2}+b h_{2}^{3}\right) \partial_{x} p_{2}\right)=0 .
\end{gathered}
$$

with

$$
\partial_{x} p_{2}=\rho_{2} g \partial_{x}\left(h_{1}+h_{2}\right) \quad \text { and } \quad V\left(h_{1}\right)=\frac{1}{h_{1}^{3}}-\frac{\alpha}{h_{1}^{4}} \quad(\alpha>0)
$$

where $(t, x) \in(0, T) \times[0,1]$.

These equations represent a system composed of two layers of immiscible fluids. 
We denote $h_{1}, h_{2}$ respectively, the water and the pollutant heights, $u_{1}$ is the water velocity. We introduce the ratio of densities $r=\frac{\rho_{2}}{\rho_{1}}$ where $\rho_{1}$ and $\rho_{2}$ denoted respectively the densities of the water and the pollutant. $v_{1}$ is the kinematic viscosity; $g$ is the constant gravity.

The coefficients $\sigma, r_{1}$ and $\beta$ are respectively the coefficients of the interfaz tension, quadratic friction and positive slip length parameters. $a$ and $b$ are respectively depending on the friction at the interfaz and coefficient of the viscosity of the pollutant. $\alpha$ and $\varepsilon$ are positive constants.

From a theoretical point of view, several studies have been carried out on the existence of global weak solutoins of shallow-water equations model. We cite among others some results concerning the existence of global weak solutions of the transport models.

The existence of weak solutions for a viscous sedimentation model is obtained by assuming smallness of the data in (Toumbou, Le Roux \& Sene, 2007) in which the viscosity term is the form $-v \Delta u$. The authors in (Zabsonré Lucas \& Fern ndez-Nieto, 2009) studied the stability of global weak solutions for a sediment transport model in two- dimensional with the term $v \operatorname{div}(h \nabla u)$.

The stability result is obtained without any restriction on the data and by using a mathematical entropy introduced first in (Bresch \& Desjardins, 2003) namely BD entropy. The BD entropy allows us to get regularity on $\partial_{x} \sqrt{h}$, see lemma 2 . The authors in (Bresch, Desjardins \& Grard-Varet, 2007; Bresch, Desjardins \& Lin, 2003; Marche, 2007; Mellet \& Vasseur, 2007) use the BD entropy inequality to get the existence results of global weak solutions for Shallow-Water and viscous compressible Navier-Stokes equations. In (Kitavtsev, Laurençot \& Niethammer, 2011) the authors proved the existence of global weak solutions for one-dimensional lubrication models that describe the dewetting process of nanoscopic thin polymer films on hy-drophobyzed substrates and take into account a large slippage at the polymer-substrate interface. In their work, the authors have used an intermolecular force that is very crucial in ours.

In the analysis, our contribution is to show the existence of global weak solutions for a similar model studied in (Roamba, Zabsonré \& Zongo, 2017) taking into acount the presence of intermolecular forces, namely long-range attractive van der Waals and short-range Born repulsive intermolecular forces without any hypothesis on unknown.

We complete the system studied with the initial conditions

$$
\begin{gathered}
h_{1}(0, x)=h_{1_{0}}(x), \quad h_{2}(0, x)=h_{2_{0}}(x), \quad\left(h_{1} u_{1}\right)(0, x)=\mathbf{m}_{0}(x) \quad \text { in }(0,1) . \\
h_{1_{0}} \in L^{2}(0,1), \quad h_{1_{0}}+h_{2_{0}} \in L^{2}(0,1), \quad \partial_{x}\left(h_{1_{0}}\right) \in L^{2}(0,1), \\
\partial_{x} \mathbf{m}_{0} \in L^{1}(0,1), \quad \mathbf{m}_{0}=0 \quad \text { if } \quad h_{1_{0}}=0, \\
\frac{\left|\mathbf{m}_{0}\right|^{2}}{h_{1_{0}}} \in L^{1}(0,1), \quad \varphi\left(h_{1_{0}}\right) \in L^{1}(0,1) .
\end{gathered}
$$

where $\varphi$ will be defined later on (see (13)).

Our paper is organized as follows. First of all, we will give in the Section 2, the definition of global weak solutions, secondly, we will establish a classical energy equality and the "mathematical BD entropy", which entails some regularities on the unknown. Thirdly, we will give a proposition allowing us to limit inferiorly the height of water which is very fundamental for the continuation since this limit study gives us additional regularities on the unknowns. Fourthly, we will give an existence theorem of global weak solutions. And finally, we will give the proof of existence Theorem including the limits passage.

\section{Main Results}

Definition 1. We say that $\left(h_{1}, h_{2}, u_{1}\right)$ is a weak solution of (1)-(3), with the initial condition verifying the entropy inequality (12) for all smooth test functions $\phi=\phi(t, x)$ with $\phi(T)=$,0 , we have:

$$
\begin{gathered}
h_{01} \phi(0, .)-\int_{0}^{T} \int_{0}^{1} h_{1} \partial_{t} \phi-\int_{0}^{T} \int_{0}^{1} h_{1} u_{1} \partial_{x} \phi=0 \\
-h_{02} \phi(0, .)-\int_{0}^{T} \int_{0}^{1} h_{2} \partial_{t} \phi-\int_{0}^{T} \int_{0}^{1} h_{2} u_{1} \partial_{x} \phi+\varepsilon \int_{0}^{T} \int_{0}^{1} \partial_{x} h_{2} \partial_{x} \phi \\
+\int_{0}^{T} \int_{0}^{1}\left(\left(a h_{2}{ }^{2}+b h_{2}{ }^{3}\right) \partial_{x} p_{2}\right) \partial_{x} \phi=0
\end{gathered}
$$




$$
\begin{gathered}
h_{01} u_{01} \phi(0, .)-\int_{0}^{T} \int_{0}^{1} h_{1} u_{1} \partial_{t} \phi-\int_{0}^{T} \int_{0}^{1} h_{1} u_{1}{ }^{2} \partial_{x} \phi+4 v_{1} \int_{0}^{T} \int_{0}^{1} h_{1} \partial_{x} u_{1} \partial_{x} \phi \\
+\frac{1}{\beta} \int_{0}^{T} \int_{0}^{1} u_{1} \phi+\int_{0}^{T} \int_{0}^{1}\left(\sigma \partial_{x}^{2} h_{1}-V\left(h_{1}\right)\right) \phi \partial_{x} h_{1}+\int_{0}^{T} \int_{0}^{1}\left(\sigma \partial_{x}^{2} h_{1}-V\left(h_{1}\right)\right) h_{1} \partial_{x} \phi \\
-\frac{1}{2} g \int_{0}^{T} \int_{0}^{1} h_{1}^{2} \partial_{x} \phi-r g \int_{0}^{T} \int_{0}^{1} h_{2} h_{1} \partial_{x} \phi+r_{1} \int_{0}^{T} \int_{0}^{1} h_{1}\left|u_{1}\right|^{2} u_{1} \phi \\
-r g \int_{0}^{T} \int_{0}^{1} \phi h_{2} \partial_{x} h_{1}-r g \int_{0}^{T} \int_{0}^{1}\left(h_{1}+h_{2}\right) h_{2} \partial_{x} \phi-r g \int_{0}^{T} \int_{0}^{1}\left(h_{1}+h_{2}\right) \partial_{x} h_{2} \phi=0
\end{gathered}
$$

Lemma 1. (Energy inequality) For classical solutions of the system (1)-(3), the following inequality holds

$$
\begin{aligned}
& \frac{d}{d t} \int_{0}^{1}\left[\frac{1}{2} h_{1}\left|u_{1}\right|^{2}+U\left(h_{1}\right)+\frac{1}{2} g(1-r)\left|h_{1}\right|^{2}+\frac{1}{2} r g\left|h_{1}+h_{2}\right|^{2}+\frac{1}{2} \sigma\left|\partial_{x} h_{1}\right|^{2}\right] \\
& +4 v_{1} \int_{0}^{1} h_{1}\left|\partial_{x} u_{1}\right|^{2}+\frac{1}{\beta} \int_{0}^{1}\left|u_{1}\right|^{2}+\frac{1}{2} g r \varepsilon \int_{0}^{1}\left|\partial_{x} h_{2}\right|^{2} \\
& +r_{1} \int_{0}^{T} \int_{0}^{1} h_{1}\left|u_{1}\right|^{4}+\rho_{2} r g^{2} \int_{0}^{1} h_{2}^{2}\left|\partial_{x}\left(h_{1}+h_{2}\right)\right|^{2}\left(a+b h_{2}\right) \leq \frac{1}{2} r g \varepsilon \int_{0}^{1}\left|\partial_{x} h_{1}\right|^{2}
\end{aligned}
$$

where the potential function $U$ is the indefinite integral of $V$ defined by $U\left(h_{1}\right)=-\frac{1}{2 h_{1}^{2}}+\frac{\alpha}{3 h_{1}^{3}}, \quad h_{1}>0$.

Proof: First, we multiply the momentum equation by $u_{1}$ and we integrate from 0 to 1 . We use the mass conservation equation of the first layer for simplification. Then, we obtain

$$
\begin{gathered}
\int_{0}^{1} \frac{1}{2} \partial_{t}\left(h_{1} u_{1}^{2}\right)+\frac{1}{2} \int_{0}^{1} g \partial_{x} h_{1}^{2} u_{1}-4 \int_{0}^{1} \partial_{x}\left(v_{1} h_{1} \partial_{x} u_{1}\right) u_{1}-\int_{0}^{1} h_{1} u_{1} \partial_{x}\left(\sigma \partial_{x}^{2} h_{1}-V\left(h_{1}\right)\right)+\int_{0}^{1} \frac{u_{1}^{2}}{\beta} \\
+r g \int_{0}^{1} h_{1} \partial_{x} h_{2} u_{1}+r g \int_{0}^{1} h_{2} \partial_{x}\left(h_{1}+h_{2}\right) u_{1}+r_{1} \int_{0}^{T} \int_{0}^{1} h_{1}\left|u_{1}\right|^{4}=0
\end{gathered}
$$

Now, let us simplify each term:

$$
\begin{aligned}
&-4 \int_{0}^{1} \partial_{x}\left(v_{1} h_{1} \partial_{x} u_{1}\right) u_{1}=4 v_{1} \int_{0}^{1} h_{1}\left(\partial_{x} u_{1}\right)^{2} \\
&-\int_{0}^{1} h_{1} u_{1} \partial_{x}\left(\sigma \partial_{x}^{2} h-V\left(h_{1}\right)\right)= \int_{0}^{1} \partial_{x}(h u)\left(\sigma \partial_{x}^{2} h_{1}-V\left(h_{1}\right)\right) \\
&=-\int_{0}^{1} \partial_{t} h_{1}\left(\sigma \partial_{x}^{2} h_{1}-V\left(h_{1}\right)\right) \\
&=\int_{0}^{1} \sigma \partial_{x t} h_{1} \partial_{x} h_{1}+\int_{0}^{1} \partial_{t}\left(U\left(h_{1}\right)\right) \\
&=\int_{0}^{1} \partial_{t}\left(\frac{1}{2} \sigma\left|\partial_{x} h_{1}\right|^{2}+U\left(h_{1}\right)\right)
\end{aligned}
$$

- $r g \int_{0}^{1} h_{1} \partial_{x} h_{2} u_{1}=-r g \int_{0}^{1} h_{2} \partial_{x}\left(h_{1} u_{1}\right)=r g \int_{0}^{1} h_{2} \partial_{t} h_{1}$

- $\frac{1}{2} g \int_{0}^{1} \partial_{x} h_{1}^{2} u_{1}=\frac{1}{2} g \frac{d}{d t} \int_{0}^{1}\left|h_{1}\right|^{2}$

- $r g \int_{0}^{1} h_{2} \partial_{x}\left(h_{1}+h_{2}\right) u_{1}=-r g \int_{0}^{1}\left(h_{1}+h_{2}\right) \partial_{x}\left(h_{2} u_{1}\right)$

The equation for the thin film flow gives us : $\partial_{x}\left(h_{2} u_{1}\right)=-\partial_{t} h_{2}+\varepsilon \partial_{x}^{2} h_{2}+\partial_{x}\left(\left(a h_{2}{ }^{2}+b h_{2}{ }^{3}\right) \partial_{x} p_{2}\right)$ and we have:

- $r g \int_{0}^{1} h_{2} \partial_{x}\left(h_{1}+h_{2}\right) u_{1}=r g \varepsilon \int_{0}^{1} \partial_{x} h_{1} \partial_{x} h_{2}+r g \varepsilon \int_{0}^{1}\left|\partial_{x} h_{2}\right|^{2}+\frac{1}{2} r g \frac{d}{d t} \int_{0}^{1}\left|h_{2}\right|^{2}$

Substituting all these terms in (11), we get (10) by integrating under 0 to $T$.

$$
+\rho_{2} r g^{2} \int_{0}^{1} h_{2}^{2}\left|\partial_{x}\left(h_{1}+h_{2}\right)\right|^{2}\left(a+b h_{2}\right)+r g \int_{0}^{1} h_{1} \partial_{t} h_{2}
$$


Remark 1. 1. Notice that the term in the right of (10) can be controlled using Gronwall's lemma.

2. The term $\int_{0}^{1} \int_{0}^{T} U\left(h_{1}\right)\left|\partial_{x} h_{1}\right|^{2}$ can be absorbed by $\int_{0}^{1} \int_{0}^{T}\left(\frac{\alpha}{6 h_{1}^{3}}-\frac{2}{3 \alpha^{2}}\right)\left|\partial_{x} h_{1}\right|^{2}$ thanks to the work done in (Kitavtsev, Laurençot $\mathcal{E}$ Niethammer, 2011).

Corollary 1. Let $\left(h_{1}, h_{2}, u_{1}\right)$ be a solution of model $(1)-(2)$. Then, thanks to Lemma 1 we have:

$$
\begin{aligned}
& h_{1} \quad \text { is bounded in } \quad L^{\infty}\left(0, T ; L^{2}(0,1)\right) \text {, } \\
& \partial_{x} h_{1} \quad \text { is bounded in } \quad L^{\infty}\left(0, T ; L^{2}(0,1)\right) \text {, } \\
& \left(h_{1}+h_{2}\right) \quad \text { is bounded in } \quad L^{\infty}\left(0, T ; L^{2}(0,1)\right) \text {, } \\
& \sqrt{h_{1}} \partial_{x} u_{1} \quad \text { is bounded in } \quad L^{2}\left(0, T ; L^{2}(0,1)\right) \text {, } \\
& u_{1} \quad \text { is bounded in } \quad L^{2}\left(0, T ; L^{2}(0,1)\right) \text {, } \\
& h_{2} \sqrt{a+b h_{2}}\left(\partial_{x}\left(h_{1}+h_{2}\right)\right) \quad \text { is bounded in } \quad L^{2}\left(0, T ; L^{2}(0,1)\right) \text {, } \\
& h_{1}^{-1} \quad \text { is bounded in } \quad L^{\infty}\left(0, T ; L^{2}(0,1)\right) \text {, } \\
& h_{1}^{-\frac{3}{2}} \quad \text { is bounded in } \quad L^{\infty}\left(0, T ; L^{2}(0,1)\right) \text {, } \\
& \partial_{x} h_{2} \quad \text { is bounded in } \quad L^{2}\left(0, T ; L^{2}(0,1)\right) \text {, } \\
& \sqrt{h_{1}}\left|u_{1}\right|^{2} \quad \text { is bounded in } \quad L^{2}\left(0, T ; L^{2}(0,1)\right) \text {. }
\end{aligned}
$$

We will need furthermore, some additional regularities on $h_{1}$ and this will be achieved through an additional BD entropy inequality presented in the next lemma.

Lemma 2. For smooth solutions $\left(h_{1}, h_{2}, u_{1}\right)$ of model (1) - (3) satisfying the classical energy equality of the Lemma 1, we have the following mathematical BD entropy inequality:

$$
\begin{aligned}
& \frac{d}{d t} \int_{0}^{1}\left[\frac{1}{2} h_{1}\left|u_{1}+\partial_{x} \varphi\left(h_{1}\right)\right|^{2}-\frac{1}{\beta} \varphi\left(h_{1}\right)+\frac{1}{2} g(1-r)\left|h_{1}\right|^{2}+\frac{1}{2} r g\left|h_{1}+h_{2}\right|^{2}+\frac{1}{2} \sigma\left|\partial_{x} h_{1}\right|^{2}+U\left(h_{1}\right)\right] \\
+ & \frac{1}{\beta} \int_{0}^{1}\left|u_{1}\right|^{2}+4 v_{1} \int_{0}^{1}\left(g+g r \frac{h_{2}}{h_{1}}+V^{\prime}\left(h_{1}\right)\right)\left|\partial_{x} h_{1}\right|^{2}+r g \int_{0}^{1}\left(\varepsilon+4 v_{1} \frac{h_{2}}{h_{1}}\right) \partial_{x} h_{1} \partial_{x} h_{2}+4 v_{1} \sigma \int_{0}^{1}\left|\partial_{x}^{2} h_{1}\right|^{2} \\
+ & 4 v_{1} r_{1} \int_{0}^{T} \int_{0}^{1}\left|u_{1}\right|^{2} u_{1} \partial_{x} h_{1}+g r \varepsilon \int_{0}^{1}\left|\partial_{x} h_{2}\right|^{2}+r g^{2} \int_{0}^{1} h_{2}^{2}\left(a+b h_{2}\right)\left(\partial_{x}\left(h_{1}+h_{2}\right)\right)^{2} \leq \frac{1}{2} r g \varepsilon \int_{0}^{1}\left|\partial_{x} h_{1}\right|^{2}
\end{aligned}
$$

where

$$
\varphi\left(h_{1}\right)=4 v_{1} \log h_{1}
$$

We need these results to prove the above lemma.

Proposition 1. If $h_{1}$ has the regularities established in corollary 2.1, then there exists constants $c_{1}$ and $c_{2}$ such as $0<$ $c_{1}<h_{1}<c_{2}$.

Proof: We follow the lines performed in (Kitavtsev, Laurençot $\&$ Niethammer, 2011). Using the bound on $\partial_{x} h_{1}$ we obtain:

$$
h(x, t)-h(y, t) \leq\left|\int_{x}^{y} \partial_{x} h_{1}(z, t) d z\right| \leq\|x-y\|^{1 / 2}\left\|\partial_{x} h_{1}(t)\right\|_{2} \leq \frac{C_{1}}{\sqrt{\sigma}}|x-y|^{1 / 2}
$$

for all $(x, y) \in(0,1) \times(0,1)$ and $t \in(0, T)$. Next we integrate the above inequality with respect to $y \in(0,1)$, readily give the upper bound. To establish the lower bound for $h_{1}$, we combine the $L^{\infty}\left(0, T ; L^{2}(0,1)\right)$-estimates on $h_{1}^{-3 / 2}$ and $\sqrt{\sigma} \partial_{x} h_{1}$ just established to obtain a bound on the norm of $1 / \sqrt{h_{1}}$ in $L^{\infty}\left(0, T ; W^{1,1}(0,1)\right)$ since

$$
\int_{0}^{1}\left|\partial_{x}\left(h_{1}^{-1 / 2}\right)\right|=\frac{1}{2} \int_{0}^{1} \frac{\left|\partial_{x} h_{1}\right|}{h_{1}^{3 / 2}} \leq \frac{1}{2 \sqrt{\sigma}}\left\|\sqrt{\sigma} \partial_{x} h_{1}\right\|_{2}\left\|h_{1}^{-3 / 2}\right\|_{2}
$$

Due to the continuous embedding of $W^{1,1}(0,1)$ in $L^{\infty}(0,1)$, we get the positive lower bound. 
Proof: (Lemma 2) Let us multiply the equation (2) by $\partial_{x} \varphi\left(h_{1}\right)$, integrate with respect to $x$ and use an integration by parts, and using (1), we have:

$$
\begin{gathered}
4 v_{1} \int_{0}^{1}\left(\partial_{t} u_{1}+u_{1} \partial_{x} u_{1}\right) \partial_{x} h_{1}+4 v_{1} g \int_{0}^{1}\left|\partial_{x} h_{1}\right|^{2}+16 v_{1}^{2} \int_{0}^{1} h_{1} \partial_{x} u_{1} \partial_{x}\left(\frac{\partial_{x} h_{1}}{h_{1}}\right)+4 v_{1} \int_{0}^{1} \frac{u_{1} \partial_{x} h_{1}}{\beta h_{1}}+4 v_{1} \sigma \int_{0}^{1}\left|\partial_{x}^{2} h_{1}\right|^{2} \\
+4 v_{1} \int_{0}^{1} V^{\prime}\left(h_{1}\right)\left|\partial_{x} h_{1}\right|^{2}+4 v_{1} r g \int_{0}^{1} \partial_{x} h_{2} \partial_{x} h_{1}+4 v_{1} r g \int_{0}^{1} \frac{h_{2}}{h_{1}}\left|\partial_{x} h_{1}\right|^{2}+4 v_{1} r g \int_{0}^{1} \frac{h_{2}}{h_{1}} \partial_{x} h_{2} \partial_{x} h_{1}=0 .
\end{gathered}
$$

On the one hand, a further integration by parts of the first integral of (14), equation (1), and the energy equality (10) give

$$
\begin{aligned}
& 4 v_{1} \int_{0}^{1}\left(\partial_{t} u_{1}+u_{1} \partial_{x} u_{1}\right) \partial_{x} h_{1} \\
& =4 v_{1}\left(\frac{d}{d t} \int_{0}^{1} u_{1} \partial_{x} h_{1}-\int_{0}^{1} u_{1} \partial_{x t}^{2} h_{1}+\int_{0}^{1} u_{1} \partial_{x} u_{1} \partial_{x} h_{1}\right) \\
& =4 v_{1}\left(\frac{d}{d t} \int_{0}^{1} u_{1} \partial_{x} h_{1}-\int_{0}^{1} \partial_{x} u_{1} \partial_{x}\left(h_{1} u_{1}\right)+\int_{0}^{1} u_{1} \partial_{x} u_{1} \partial_{x} h_{1}\right) \\
& =4 v_{1}\left(\frac{d}{d t} \int_{0}^{1} u_{1} \partial_{x} h_{1}-\int_{0}^{1} h_{1}\left(\partial_{x} u_{1}\right)^{2}\right) \\
& =\frac{d}{d t} \int_{0}^{1}\left[4 v_{1} u_{1} \partial_{x} h_{1}+\frac{1}{2} h_{1}\left|u_{1}\right|^{2}+U\left(h_{1}\right)+\frac{1}{2} g(1-r)\left|h_{1}\right|^{2}+\frac{1}{2} r g\left|h_{1}+h_{2}\right|^{2}+\frac{1}{2} \sigma\left|\partial_{x} h_{1}\right|^{2}\right] \\
& +\frac{1}{\beta} \int_{0}^{1}\left|u_{1}\right|^{2}+r g \varepsilon \int_{0}^{1} \partial_{x} h_{1} \partial_{x} h_{2}+r g \varepsilon \int_{0}^{1}\left|\partial_{x} h_{2}\right|^{2}+r g^{2} \int_{0}^{1} h_{2}^{2}\left(a+b h_{2}\right)\left(\partial_{x}\left(h_{1}+h_{2}\right)\right)^{2}
\end{aligned}
$$

On the other hand,we can write the third and the fourth integrals of (14) as

$$
\begin{aligned}
&-16 v_{1}^{2} \int_{0}^{1} \partial_{x}\left(\frac{\partial_{x} h_{1}}{h_{1}}\right) \partial_{x} u_{1} h_{1}=\frac{1}{2} \frac{d}{d t} \int_{0}^{1} h_{1}\left|\varphi\left(h_{1}\right)\right|^{2} \\
& \text { - } 4 v_{1} \int_{0}^{1} \frac{u_{1} \partial_{x} h_{1}}{\beta h_{1}}=-4 v_{1} \int_{0}^{1} \frac{\partial_{x}\left(u_{1} h_{1}\right)}{\beta h_{1}}+4 v_{1} \int_{0}^{1} \frac{\partial_{x} u_{1}}{\beta} \\
&=-\frac{1}{\beta} \frac{d}{d t} \int_{0}^{1} \varphi\left(h_{1}\right) .
\end{aligned}
$$

(See (Kitavtsev, Laurençot \& Niethammer, 2011)).

Substituting finally the last three identities into (14), we obtain (13).

\section{Remark 2.}

In Lemma 2 all the terms, excepted

$$
\int_{0}^{T} \int_{0}^{1}\left|u_{1}\right|^{2} u_{1} \partial_{x} h_{1}, \quad \int_{0}^{T} \int_{0}^{1}\left(\varepsilon+4 v_{1} \frac{h_{2}}{h_{1}}\right) \partial_{x} h_{1} \partial_{x} h_{2} \quad \text { and } \int_{0}^{1} \int_{0}^{T} V^{\prime}\left(h_{1}\right)\left|\partial_{x} h_{1}\right|^{2}
$$

are controlled since they have the good sign. The control of the term $\int_{0}^{T} \int_{0}^{1}\left|u_{1}\right|^{2} u_{1} \partial_{x} h_{1}$ takes inspiration in (Roamba, Zabsonr?E Traor? 2016). The term $\int_{0}^{1} \int_{0}^{T} V^{\prime}\left(h_{1}\right)\left|\partial_{x} h_{1}\right|^{2}$ can be absorbed thanks to the work done in (Kitavtsev, Laurençot $\mathcal{E}$ Niethammer, 2011). It remains for us to control the term $\int_{0}^{T} \int_{0}^{1}\left(\varepsilon+4 v_{1} \frac{h_{2}}{h_{1}}\right) \partial_{x} h_{1} \partial_{x} h_{2}$.

Proposition 2. There exists a constant $C$ such as $\left\|\left(\varepsilon+4 v_{1} \frac{h_{2}}{h_{1}}\right) \partial_{x} h_{1} \partial_{x} h_{2}\right\|_{L^{2}\left(0, T ; L^{2}(0,1)\right)} \leq C$.

Proof: We have:

$\int_{0}^{T} \int_{0}^{1} \partial_{x} h_{1} \partial_{x} h_{2} \leq \frac{1}{2} \int_{0}^{T} \int_{0}^{1}\left(\left|\partial_{x} h_{1}\right|^{2}+\left|\partial_{x} h_{2}\right|^{2}\right) \leq C^{\prime}$ because $\partial_{x} h_{1}, \partial_{x} h_{2}$ are in $L^{\infty}\left(0, T ; L^{2}(0,1)\right)$. It remains for us to 
limit $\frac{h_{2}}{h_{1}} \partial_{x} h_{1} \partial_{x} h_{2}$.

Since $\frac{h_{2}}{h_{1}} \partial_{x} h_{1} \partial_{x} h_{2}=\partial_{x} \varphi\left(h_{1}\right) \partial_{x} h_{1} \partial_{x} h_{2}$, we can write:

$\int_{0}^{T} \int_{0}^{1}\left|\frac{h_{2}}{h_{1}} \partial_{x} h_{1} \partial_{x} h_{2}\right| \leq \frac{1}{2} \int_{0}^{T} \int_{0}^{1}\left|\partial_{x} \varphi\left(h_{1}\right)\right|^{2}+\frac{1}{2} \int_{0}^{T} \int_{0}^{1}\left|h_{2} \partial_{x} h_{2}\right|^{2}$.

We will now look at the two terms to the right of the above inequality separately. For the first one, we have:

$\int_{0}^{T} \int_{0}^{1}\left|\partial_{x} \varphi\left(h_{1}\right)\right|^{2}=\int_{0}^{T} \int_{0}^{1} \frac{\left|\partial_{x} h_{1}\right|^{2}}{h_{1}^{2}} \leq \int_{0}^{T} \int_{0}^{1}\left|\partial_{x} h_{1}\right|^{2} \leq \mathbf{C}\left(h_{1}\right.$ being bounded inferiorly) and $\partial_{x} h_{2}$ is in $L^{2}\left(0, T ; L^{2}(0,1)\right)$, so $\partial_{x} \varphi\left(h_{1}\right)$ is in $L^{2}\left(0, T ; L^{2}(0,1)\right)$. For the second one, since $h_{2} \in L^{\infty}\left(0, T ; H^{1}(0,1)\right)$ and $\partial_{x} h_{2} \in L^{\infty}\left(0, T ; L^{2}(0,1)\right)$ then, $h_{2} \partial_{x} h_{2} \in L^{\infty}\left(0, T ; L^{2}(0,1)\right)$ see (Marche, 2007) for instance. Which completes the proof.

Lemma 3. For classical solutions of the system (1) - (3) with a first component $h_{1}$, we have

$$
\frac{1}{4} \int_{0}^{1} h_{1}\left|\partial_{x} \varphi\left(h_{1}\right)\right|^{2} \leq \frac{1}{2} \int_{0}^{1} h_{1}\left(u+\partial_{x} \varphi\left(h_{1}\right)\right)^{2}+2 E\left(h_{1}, h_{2}, u_{1}\right)+\frac{1}{3 \alpha^{2}}
$$

with

$E\left(h_{1}, h_{2}, u_{1}\right)=\int_{0}^{1}\left[\frac{1}{2} h_{1}\left|u_{1}\right|^{2}+U\left(h_{1}\right)+\left.\frac{1}{2} r g\left|h_{1}+\frac{1}{2} g(1-r)\right| h_{1}\right|^{2}+\left.h_{2}\right|^{2}+\frac{1}{2} \sigma\left|\partial_{x} h_{1}\right|^{2}\right]$.

Proof: Using the elementary inequality

$$
(y+z)^{2} \geq \frac{y^{2}}{2}-z^{2}
$$

the fact that

$$
U\left(h_{1}\right) \geq \frac{-1}{6 \alpha^{2}}
$$

for $h_{1}>0$, and the definition of $E$, we obtain:

$$
\begin{aligned}
& \int_{0}^{1} h_{1}\left(u_{1}+\partial_{x} \varphi\left(h_{1}\right)\right)^{2} \geq \frac{1}{2} \int_{0}^{1} h_{1}\left|\partial_{x} \varphi\left(h_{1}\right)\right|^{2}-\int_{0}^{1} h_{1} u_{1}^{2} \\
& \geq \frac{1}{2} \int_{0}^{1} h_{1}\left|\partial_{x} \varphi\left(h_{1}\right)\right|^{2}-2\left[E\left(h_{1}, h_{2}, u_{1}\right)-\int_{0}^{1}\left(U\left(h_{1}\right)+\frac{1}{2} g(1-r)\left|h_{1}\right|^{2}+\frac{1}{2} r g\left|h_{1}+h_{2}\right|^{2}+\frac{1}{2} \sigma\left|\partial_{x} h_{1}\right|^{2}\right)\right] \\
& \geq \frac{1}{2} \int_{0}^{1} h_{1}\left|\partial_{x} \varphi\left(h_{1}\right)\right|^{2}-2 E\left(h_{1}, h_{2}, u_{1}\right)-\frac{1}{3 \alpha^{2}} \\
& \text { from that the statement of the lemma follows. }
\end{aligned}
$$

Corollary 2. Let $\left(h_{1}, h_{2}, u_{1}\right)$ be a solution of model (1) - (3).

Then, thanks to Lemma 2 and Lemma 3 we have:

$$
\begin{array}{rrr}
\sqrt{h_{1}} & \text { is bounded in } & L^{\infty}\left(0, T ; L^{2}(0,1)\right), \\
\partial_{x} \sqrt{h_{1}} & \text { is bounded in } & L^{\infty}\left(0, T ; L^{2}(0,1)\right), \\
\partial_{x}^{2} h_{1} & \text { is bounded in } & L^{2}\left(0, T ; L^{2}(0,1)\right) .
\end{array}
$$

Remark 3. 1. In the Corollary 1, the estimate

$$
\sqrt{h_{1}} u_{1} \quad \text { is bounded in } \quad L^{\infty}\left(0, T ; L^{2}(0,1)\right)
$$

implies,

$$
h_{1} u_{1} \quad \text { is bounded in } \quad L^{\infty}\left(0, T ; L^{2}(0,1)\right)
$$

this leads us

$$
\partial_{t} h_{1} \quad \text { is bounded in } \quad L^{\infty}\left(0, T ; W^{-1,2}(0,1)\right) .
$$

2. We have the additional regularities thanks to Corollary 1:

(a) $h_{1}$ is bounded in $L^{2}\left(0, T ; H^{1}(0,1)\right)$, 
(b) $h_{1} u_{1}$ is bounded in $L^{3}\left(0, T ; L^{3}(0,1)\right) \cap L^{\infty}\left(0, T ; L^{2}(0,1)\right) \cap L^{2}\left(0, T ; W^{1,1}(0,1)\right)$.

Remark 4. We have the following additional regularities:

1. $h_{1}$ and $h_{2}$ are bounded in $L^{\infty}\left(0, T ; L^{2}(0,1)\right)$.

2. $\sqrt{h_{1}}$ is bounded in $L^{2}\left(0, T ; H^{1}(0,1)\right)$.

Indeed,

by integrating the mass equation, we obtain directly $\sqrt{h_{1}^{k}}$ in $L^{\infty}\left(0, T ; L^{2}(\Omega)\right)$. As Corollary 2 gives us $\partial_{x} \sqrt{h_{1}^{k}}$ in $L^{\infty}\left(0, T ; L^{2}(\Omega)\right)$, so $\sqrt{h_{1}^{k}}$ is bounded in $L^{\infty}\left(0, T ; H^{1}(\Omega)\right)$.

Theorem 1. There exists a global weak solutions to the system (1)-(3) with initial data (5), (6) and satisfying energy equality (10) and energy inequality (12).

\section{Convergences}

The objective of this section is to prove the Theorem 1 . Let $\left(h_{1}^{k}, h_{2}^{k}, u_{1}^{k}\right)$ be a sequence of weak solutions with initial data

$$
h_{1 \mid t=0}^{k}=h_{1_{0}}^{k}, \quad h_{2 \mid t=0}^{k}=h_{2_{0}}^{k}, \quad\left(h_{1}^{k} u^{k}\right)_{\mid t=0}=m_{0}^{k}
$$

such as

$$
h_{1_{0}}^{k} \longrightarrow h_{1_{0}} \text { in } L^{1}(\Omega), \quad h_{2_{0}}^{k} \longrightarrow h_{2_{0}} \text { in } L^{1}(\Omega), \quad m_{0}^{k} \longrightarrow m_{0} \text { in }\left(L^{1}(\Omega)\right)^{2}
$$

and satisfies

$$
-\frac{1}{\beta} \int_{0}^{1} \varphi\left(h_{1_{0}}^{k}\right)+\int_{0}^{1}\left[h_{1_{0}}^{k}\left|u_{1_{0}}^{k}\right|^{2}+64 v_{1}^{2}\left|\partial_{x} \sqrt{h_{1_{0}}^{k}}\right|^{2}+\frac{1}{2} g(1-r)\left|h_{1_{0}}^{k}\right|^{2}+\frac{1}{2} r g\left|h_{1_{0}}^{k}+h_{2_{0}}^{k}\right|^{2}+\frac{1}{2} \sigma\left|\partial_{x} h_{1_{0}}^{k}\right|^{2}\right] \leq C .
$$

Such approximate solutions can be built by a regularization of capillary effect.

3.1 Strong Convergence of $\sqrt{h_{1}^{k}}, \quad h_{1}^{k}$ and $h_{2}^{k}$

From the remark 4:

$$
\sqrt{h_{1}^{k}} \text { is bounded in } L^{\infty}\left(0, T ; H^{1}(\Omega)\right) .
$$

Moreover, using the mass equation, we obtain the following equality:

$$
\partial_{t} \sqrt{h_{1}^{k}}=\frac{1}{2} \sqrt{h_{1}^{k}} \partial_{x} u_{1}^{k}-\partial_{x}\left(\sqrt{h_{1}^{k}} u_{1}^{k}\right)
$$

which gives that $\partial_{t} \sqrt{h_{1}^{k}}$ is bounded in $L^{2}\left(0, T ; H^{-1}(\Omega)\right)$.

Applying Aubin-Simon lemma (see (Lions, 1969), (Simon, 1987)), we can extract a subsequence, still denoted $\left(h_{1}^{k}\right)_{1 \leq k}$, such as

$$
\sqrt{h_{1}^{k}} \text { converges strongly to } \sqrt{h_{1}} \text { in } C^{0}\left(0, T ; L^{2}(0,1)\right) \text {. }
$$

According to the Proposition 1, we show that

$$
\left|h_{1}^{k}-h_{1}\right| \leq \sqrt{c_{2}}\left|\sqrt{h_{1}^{k}}-\sqrt{h_{1}}\right| \Rightarrow\left|h_{1}^{k}-h_{1}\right|^{2} \leq c_{2}\left|\sqrt{h_{1}^{k}}-\sqrt{h_{1}}\right|^{2} .
$$

This ensures

$$
h_{1}^{k} \quad \text { converges strongly to } h_{1} \text { in } L^{2}\left(0, T ; L^{2}(0,1)\right) \text {. }
$$

We have $h_{2}^{k}$ bounded in $L^{2}\left(0, T ; H^{1}(0,1)\right)$. Moreover, we have $\partial_{t} h_{2}{ }^{k}=-\partial_{x}\left(h_{2}^{h} u_{1}^{k}\right)+\varepsilon \partial_{x}^{2} h_{2}$.

Since $h_{2}^{k}$ is in $L^{\infty}\left(0, T ; L^{2}(0,1)\right)$ and $u_{1}^{k}$ is in $L^{2}\left(0, T ; L^{2}(0,1)\right)$, we show that the first term is in $L^{2}\left(0, T ; W^{-1,1}(0,1)\right)$. By analogy we prove that the last term is in the same space and we also get $\partial_{t} h_{2}^{k}$ in this space. According to the Aubin-Simon lemma, we have:

$$
h_{2}^{k} \text { converges strongly to } h_{2} \text { in } L^{2}\left(0, T ; W^{-1,1}(0,1)\right) .
$$




\subsection{Strong Convergence of $h_{1}^{k} u_{1}^{k}$}

We have $h_{1}^{k} u_{1}^{k}=\sqrt{h_{1}^{k}} \sqrt{h_{1}^{k}} u_{1}^{k}$. Since $\sqrt{h_{1}^{k}}$ is bounded in $L^{\infty}\left(0, T ; L^{4}(0,1)\right)$ and $\sqrt{h_{1}^{k}} u_{1}^{k}$ is bounded in $L^{\infty}\left(0, T ; L^{2}(0,1)\right)$ so $h_{1}^{k} u_{1}^{k}$ bounded in $L^{\infty}\left(0, T ; L^{\frac{4}{3}}(0,1)\right)$. Writingthe gradient as follows:

$$
\partial_{x}\left(h_{1}^{k} u_{1}^{k}\right)=h_{1}^{k} \partial_{x} u_{1}^{k}+u_{1}^{k} \partial_{x} h_{1}^{k}=\sqrt{h_{1}^{k}} \sqrt{h_{1}^{k}} \partial_{x} u_{1}^{k}+u_{1}^{k} \partial_{x} h_{1}^{k}
$$

since the first term is in $L^{2}\left(0, T ; L^{\frac{4}{3}}(0,1)\right)$ and thanks to the Corollary 1 , the second one belongs to $L^{\infty}\left(0, T ; W^{-1, \frac{4}{3}}(0,1)\right) \cap$ $L^{2}\left(0, T ; L^{1}(0,1)\right)$, we have

$$
\left(h_{1}^{k} u_{1}^{k}\right)_{k} \text { bounded in } L^{2}\left(0, T ; W^{1,1}(0,1)\right)
$$

Moreover, the momentum equation (2) enables us to write the time derivation of the water discharge:

$$
\begin{gathered}
\partial_{t}\left(h_{1}^{k} u_{1}^{k}\right)=-\partial_{x}\left(h_{1}^{k} u_{1}^{k^{2}}\right)-\frac{1}{2} g \partial_{x} h_{1}^{k^{2}}+4 v_{1} \partial_{x}\left(h_{1}^{k} \partial_{x} u_{1}\right)+\frac{u_{1}^{k}}{\beta}+h_{1}^{k} \partial_{x}\left(\sigma \partial_{x}^{2} h_{1}^{k}-V\left(h_{1}^{k}\right)\right) \\
-r g h_{1}^{k} \partial_{x} h_{2}^{k}-r g h_{2}^{k} \partial_{x}\left(h_{1}^{k}+h_{2}^{k}\right)-r_{1} h_{1}^{k}\left|u_{1}^{k}\right|^{2} u_{1}^{k} .
\end{gathered}
$$

We then study each term:

- $\partial_{x}\left(h_{1}^{k}\left(u_{1}^{k}\right)^{2}\right)=\partial_{x}\left(\sqrt{h_{1}^{k}} \sqrt{h_{1}^{k}}\left(u_{1}^{k}\right)^{2}\right)$ which is bounded in $L^{2}\left(0, T ; W^{-1, \frac{4}{3}}(0,1)\right)$.

- As $h_{1}^{k}$ is in $L^{\infty}\left(0, T ; L^{2}(0,1)\right)$, we have:

$$
\partial_{x}\left[\left(h_{1}^{k}\right)^{2}\right] \text { is in } L^{\infty}\left(0, T ; W^{-1,1}(0,1)\right) .
$$

- $\partial_{x}\left(h_{1}^{k} \partial_{x} u_{1}^{k}\right)$ is bounded in $L^{2}\left(0, T ; W^{-1, \frac{4}{3}}(0,1)\right)$.

- Let us write $h_{1}^{k} u_{1}^{k}\left(u_{1}^{k}\right)^{2}=\sqrt{h_{1}^{k}} u_{1}^{k} \sqrt{h_{1}^{k}}\left(u_{1}^{k}\right)^{2}$, which is in $L^{2}\left(0, T ; W^{-1,1}(0,1)\right)$.

- $r g h_{1}^{k} \partial_{x} h_{2}^{k}$ is bounded in $L^{2}\left(0, T ; W^{-1,1}(0,1)\right)$.

- $r g h_{2}^{k} \partial_{x}\left(h_{1}^{k}+h_{2}^{k}\right)$ is bounded in $L^{2}\left(0, T ; W^{-1,1}(0,1)\right)$.

- $\left(h_{1}^{k} \partial_{x}\left(\sigma \partial_{x}^{2} h_{1}^{k}-V\left(h_{1}^{k}\right)\right)\right)_{k}$ is bounded in $L^{2}\left(0, T ; H^{-3}(0,1)\right)$ see ( Kitavtsev, Laurençot \& Niethammer, 2011).

Thus the sequence $\partial_{t}\left(h_{1}^{k} u_{1}^{k}\right)$ is bounded in $L^{2}\left(0, T ; H^{-3}(0,1)\right)$. Then, applying Aubin-Simon lemma, we obtain,

$$
\left(h_{1}^{k} u_{1}^{k}\right)_{k} \quad \text { converges strongly to } \mathbf{m} \text { in } L^{2}\left(0, T ; L^{2}(0,1)\right) \text {. }
$$

\subsection{Strong Convergence of $\sqrt{h_{1}^{k}} u_{1}^{k}$}

Setting $\mathbf{m}^{k}=h_{1}^{k} u_{1}^{k}$, we have $\sqrt{h_{1}^{k}} u_{1}^{k}=\frac{\mathbf{m}^{k}}{\sqrt{h_{1}^{k}}}$. We want to prove the strong convergence for this term. We know that $\frac{\mathbf{m}^{k}}{\sqrt{h_{1}^{k}}}$ is bounded in $L^{\infty}\left(0, T ;\left(L^{2}(\Omega)\right)^{2}\right)$; consequently, Fatou lemma reads:

$$
\int_{\Omega} \liminf _{k} \frac{\left(\mathbf{m}^{k}\right)^{2}}{h_{1}^{k}} \leq \liminf _{k} \int_{\Omega} \frac{\left(\mathbf{m}^{k}\right)^{2}}{h_{1}^{k}}<+\infty .
$$

In particular, $\mathbf{m}$ is equal to zero for almost every $x$ where $h_{1}(t, x)$ vanishes.Then, we can define the limit velocity taking $u_{1}^{k}(t, x)=\frac{\mathbf{m}(t, x)}{h_{1}(t, x)}$ since $h_{1}(t, x) \neq 0$. So we have a link between the limits $\mathbf{m}(t, x)=h_{1}(t, x) u_{1}(t, x)$ and:

$$
\int_{\Omega} \frac{(\mathbf{m})^{2}}{h_{1}}=\int_{0}^{1} h_{1}\left|u_{1}\right|^{2}<+\infty .
$$

Moreover, we can use Fatou lemma again to write

$$
\begin{aligned}
\int_{0}^{T} \int_{\Omega} h_{1}\left|u_{1}\right|^{4} \leq \int_{0}^{T} \int_{\Omega} \liminf h_{1}\left|u_{1}\right|^{4} \leq & \liminf \int_{0}^{T} \int_{\Omega} h_{1}\left|u_{1}\right|^{4} \\
& =\liminf \int_{0}^{T} \int_{\Omega} \sqrt{h_{1}}\left|u_{1}\right|^{2} \sqrt{h_{1}}\left|u_{1}\right|^{2},
\end{aligned}
$$


which gives $\sqrt{h_{1}}\left|u_{1}\right|^{2}$ in $L^{2}\left(0, T ; L^{2}(\Omega)\right)$.

As $\mathbf{m}^{k}$ and $h_{1}^{k}$ converge almost everywhere, the sequence of $\sqrt{h_{1}^{k}} u_{1}^{k}=\frac{\mathbf{m}^{k}}{\sqrt{h_{1}^{k}}}$ converges almost everywhere to $\sqrt{h_{1}} u_{1}=\frac{\mathbf{m}}{\sqrt{h_{1}}}$. Moreover, for all $M$ positive $\sqrt{h_{1}^{k}} u_{1}^{k} 1_{\left|u_{1}^{k}\right| \leq M}$ converges to $\sqrt{h_{1}} u_{1} 1_{|u| \leq M}$ ( still assuming that $h_{1}^{k}$ does not vanish). Then, almost everywhere, we obtain the convergence of $\left(\sqrt{h_{1}^{k}} u_{1}^{k} 1_{\left|u_{1}^{k}\right| \leq M}\right)_{k}$

Finally, let us consider the following norm: $\int_{0}^{T} \int_{0}^{1}\left|\sqrt{h_{1}^{k}} u_{1}^{k}-\sqrt{h_{1}} u_{1}\right|^{2} \leq$

$$
\int_{0}^{T} \int_{0}^{1}\left(\left|\sqrt{h_{1}^{k}} u_{1}^{k} 1_{\left|u_{1}^{k}\right| \leq M}-\sqrt{h_{1}} u_{1} 1_{\left|u_{1}\right| \leq M}\right|+\left|\sqrt{h_{1}^{k}} u_{1}^{k} 1_{\left|u_{1}^{k}\right|>M}\right|+\left|\sqrt{h_{1}} u_{1} 1_{\left|u_{1}\right|>M}\right|\right)^{2}
$$

$\leq 3 \int_{0}^{T} \int_{0}^{1}\left|\sqrt{h_{1}^{k}} u_{1}^{k} 1_{\left|u_{1}^{k}\right| \leq M}-\sqrt{h_{1}} u_{1} 1_{|u| \leq M}\right|^{2}+3 \int_{0}^{T} \int_{0}^{1}\left|\sqrt{h_{1}^{k}} u_{1}^{k} 1_{\left|u_{1}^{k}\right|>M}\right|^{2}+3 \int_{0}^{T} \int_{0}^{1}\left|\sqrt{h_{1}^{k}} u_{1}^{k} 1_{\left|u_{1}^{k}\right|>M}\right|^{2}$.

Since $\sqrt{h_{1}^{k}}$ is in $L^{2}\left(0, T ; L^{4}(0,1)\right), \sqrt{h_{1}^{k}} u_{1}^{k} 1_{\left|u_{1}^{k}\right| \leq M}$ is bounded in this space. So, as we have seen previously, the first integral tends to zero. Let us study the other two terms:

$$
\int_{0}^{1}\left|\sqrt{h_{1}^{k}} u_{1}^{k} 1_{\left|u_{1}^{k}\right|>M}\right|^{2} \leq \frac{1}{M^{2}} \int_{0}^{1} h_{1}^{k}\left(u_{1}^{k}\right)^{4} \leq \frac{c}{M^{2}} \quad \text { and } \quad \int_{0}^{1}\left|\sqrt{h_{1}} u 1_{|u|>M}\right|^{2} \leq \frac{1}{M^{2}} \int_{0}^{1} h_{1} u_{1}^{4} \leq \frac{c}{M^{2}},
$$

for all $M>0$. When $\mathrm{M}$ tends to the infinity, our two integrals tend to zero. Then

$$
\sqrt{h_{1}^{k}} u_{1}^{k} \quad \text { converges strongly to } \sqrt{h_{1}} u \text { in } L^{2}\left(0, T ;\left(L^{2}(\Omega)\right)^{2}\right) .
$$

\subsection{Strong Convergence of $\partial_{x} h_{1}^{k}, \quad h_{2}^{k} \partial_{x} h_{1}^{k}, \quad \partial_{x}^{2} h_{1}^{k}, \quad h_{1}^{k} \partial_{x}^{2} h_{1}$ and $\partial_{x} h_{1}^{h} \partial_{x}^{2} h_{1}^{k}$}

- We have $\partial_{x} h_{1}$ bounded in $L^{2}\left(0, T ; H^{1}(0,1)\right)$ and $\partial_{t} \partial_{x} h_{1}$ is bounded in $L^{\infty}\left(0, T ; H^{-1}(0,1)\right)$ since $\partial_{t} h_{1}$ is bounded in $L^{\infty}\left(0, T ; H^{-1}(0,1)\right)$. Thanks to compact injection of $H^{1}(0,1)$ in $L^{2}(0,1)$ in one dimension, we have:

$$
\partial_{x} h_{1}^{k} \quad \text { converges strongly to } \quad \partial_{x} h_{1} \quad \text { in } L^{2}\left(0, T ; L^{2}(0,1)\right) .
$$

- The bound of $\partial_{x}^{2} h_{1}^{k}$ in $L^{2}\left(0, T ; L^{2}(0,1)\right)$ and $\partial_{x} h_{2}^{k}$ in $L^{2}\left(0, T ; L^{2}(0,1)\right)$ gives us:

$$
\begin{aligned}
& \partial_{x}^{2} h_{1}^{k} \quad \text { converges weakly to } \quad \partial_{x}^{2} h_{1} \quad \text { in } L^{2}\left(0, T ; L^{2}(0,1)\right), \\
& \partial_{x} h_{2}^{k} \quad \text { converges weakly to } \quad \partial_{x} h_{2} \quad \text { in } L^{2}\left(0, T ; L^{2}(0,1)\right) .
\end{aligned}
$$

- Thanks to the strong convergence of $h_{1}^{k}, h_{2}^{k}, \partial_{x} h_{1}^{k}$ and the weak convergence of $\partial_{x}^{2} h_{1}^{k}$, we have:

$$
\begin{array}{rlll}
\left(h_{2}^{k} \partial_{x} h_{1}^{k}\right)_{k} & \text { converges strongly to } & h_{2} \partial_{x} h_{1} & \text { in } L^{1}\left(0, T ; L^{1}(0,1)\right), \\
\left(h_{1}^{k} \partial_{x}^{2} h_{1}^{k}\right)_{k} & \text { converges weakly to } & h_{1} \partial_{x}^{2} h_{1} \quad \text { in } L^{1}\left(0, T ; L^{1}(0,1)\right), \\
\left(\partial_{x} h_{1}^{k} \partial_{x}^{2} h_{1}^{k}\right)_{k} & \text { converges weakly to } & \partial_{x} h_{1} \partial_{x}^{2} h_{1} \quad \text { in } L^{1}\left(0, T ; L^{1}(0,1)\right), \\
\left(h_{1}^{k} \partial_{x} h_{2}^{k}\right)_{k} & \text { converges strongly to } & h_{1} \partial_{x} h_{2} & \text { in } L^{1}\left(0, T ; L^{1}(0,1)\right), \\
\left(h_{2}^{k} \partial_{x} h_{2}^{k}\right)_{k} & \text { converges strongly to } & h_{2} \partial_{x} h_{2} & \text { in } L^{1}\left(0, T ; L^{1}(0,1)\right), \\
\left(\left(h_{1}^{k}\right)^{2}\right)_{k} & \text { converges strongly to } & h_{1}^{2} \quad \text { in } L^{1}\left(0, T ; L^{1}(0,1)\right), \\
\left(\left(h_{2}^{k}\right)^{2}\right)_{k} & \text { converges strongly to } & h_{2}^{2} \quad \text { in } L^{1}\left(0, T ; L^{1}(0,1)\right), \\
\left(h_{1}^{k} h_{2}^{k}\right)_{k} & \text { converges strongly to } & h_{1} h_{2} & \text { in } L^{1}\left(0, T ; L^{1}(0,1)\right) .
\end{array}
$$

3.5 Convergences of $h_{1}^{k} \partial_{x} u_{1}^{k}, u_{1}^{k}$ and $h_{1}^{k}\left|u_{1}^{k}\right|^{2} u_{1}^{k}$

As $u_{1}^{k}$ is bounded in $L^{2}\left(0, T ; L^{2}(0,1)\right)$, then $\partial_{x} u_{1}^{k}$ is bounded in $L^{2}\left(0, T ; W^{-1,2}(0,1)\right)$.

Then,

$$
\left(u_{1}^{k}\right)_{k} \quad \text { converges weakly to } \quad u_{1} \quad \text { in } L^{2}\left(0, T ; L^{2}(0,1)\right) \text {. }
$$


However, the function $\left(h_{1}^{k}, \partial_{x} u_{1}^{k}\right) \longmapsto h_{1}^{k} \partial_{x} u_{1}^{k}$ is a continuous in $L^{\infty}\left(0, T ; H^{1}(0,1)\right) \times L^{2}\left(0, T ; W^{-1,2}(0,1)\right)$ to $L^{2}\left(0, T ; W^{-1,2}(0,1)\right)$. So,

$$
\left(h_{1}^{k} \partial_{x} u_{1}^{k}\right)_{k} \quad \text { converges weakly to } \quad h_{1} \partial_{x} u_{1} \quad \text { in } L^{2}\left(0, T ; H^{-1}(0,1)\right) .
$$

At last, thanks to the strong convergence of $\sqrt{h_{1}^{k}} u_{1}^{k}$ in $L^{2}\left(0, T ; L^{2}(0,1)\right)$ and above the weak convergence of $u_{1}^{k}$, gives us:

$$
\left(h_{1}^{k}\left|u_{1}^{k}\right|^{2} u_{1}^{k}\right)_{k} \quad \text { converges weakly to } \quad h_{1}\left|u_{1}\right|^{2} u_{1} \quad \text { in } L^{1}\left(0, T ; L^{1}(0,1)\right) .
$$

3.6 Convergences of $\left(h_{2}^{k} u_{1}^{k}\right)_{k}$ and $\left(\partial_{x}^{2} h_{1}^{k}\right)_{k}$

We know that $\partial_{x} h_{2}^{k}$ is bounded in $L^{2}\left(0, T ; L^{2}(0,1)\right)$ this implies $\partial_{x}^{2} h_{2}^{k}$ is in $L^{1}\left(0, T ; W^{-1,2}(0,1)\right)$. So,

$$
\left(\partial_{x}^{2} h_{2}^{k}\right)_{k} \quad \text { converges weakly to } \partial_{x}^{2} h_{2} \in L^{1}\left(0, T ; W^{-1,2}(0,1)\right)
$$

To end we have $\left(u_{1}^{k}\right)_{k}$ converges weakly to $u_{1}$ in $L^{2}\left(0, T ; L^{2}(0,1)\right)$ and the strong convergence of $\left(h_{2}^{k}\right)_{k}$ to $h_{2}$, gives us:

$$
\left(h_{2}^{k} u_{1}^{k}\right)_{k} \quad \text { converges weakly to } \quad h_{2} u_{1} \quad \text { in } L^{1}\left(0, T ; L^{1}(0,1)\right) .
$$

3.7 Convergence of $\left.\left[\left(a\left(h_{2}^{k}\right)^{2}+b\left(h_{2}^{k}\right)^{3}\right)\right) \partial_{x}\left(h_{1}^{k}+h_{2}^{k}\right)\right]$

We know that $\left(\partial_{x}\left(h_{1}^{k}+h_{2}^{k}\right)\right)_{k}$ converges weakly to $\partial_{x}\left(h_{1}+h_{2}\right)$ in $L^{2}\left(0, T ; L^{2}(0,1)\right)$ and $\left(\left(a\left(h_{2}^{k}\right)^{2}+b\left(h_{2}^{k}\right)^{3}\right)\right)_{k}$ converges strongly to $a h_{2}^{2}+b h_{2}^{3}$ in $L^{1}\left(0, T ; L^{1}(0,1)\right)$.

So,

$$
\left.\left[\left(a\left(h_{2}^{k}\right)^{2}+b\left(h_{2}^{k}\right)^{3}\right)\right) \partial_{x}\left(h_{1}^{k}+h_{2}^{k}\right)\right]_{k} \quad \text { converges weakly to } \quad\left(a h_{2}^{2}+b h_{2}^{3}\right) \partial_{x}\left(h_{1}+h_{2}\right) \quad \text { in } L^{1}\left(0, T ; L^{1}(0,1)\right)
$$

3.8 Convergences of $\left(h_{1} V\left(h_{1}^{k}\right)\right)_{k}$ and $\left(V\left(h_{1}^{k}\right) \partial_{x} h_{1}^{k}\right)_{k}$

We will begin by studying the convergence of the term $h_{1} V\left(h_{1}^{k}\right)$. We have $h_{1} V\left(h_{1}^{k}\right)=\frac{1}{\left(h_{1}^{k}\right)^{2}}-\frac{\alpha}{\left(h_{1}^{k}\right)^{3}}$ and

$$
\begin{gathered}
\left|\frac{1}{\left(h_{1}^{k}\right)^{2}}-\frac{\alpha}{\left(h_{1}^{k}\right)^{3}}-\left(\frac{1}{h_{1}^{2}}-\frac{\alpha}{h_{1}^{3}}\right)\right| \leq\left|\frac{1}{\left(h_{1}^{k}\right)^{2}}-\frac{1}{h_{1}^{2}}\right|+\left|\frac{1}{\left(h_{1}^{k}\right)^{3}}-\frac{1}{h_{1}^{3}}\right| \\
\left|\frac{1}{\left(h_{1}^{k}\right)^{2}}-\frac{\alpha}{\left(h_{1}^{k}\right)^{3}}-\left(\frac{1}{h_{1}^{2}}-\frac{\alpha}{h_{1}^{3}}\right)\right| \leq \frac{\left|h_{1}^{k}-h_{1}\right|\left|h_{1}^{k}+h_{1}\right|}{\left(h_{1}^{k}\right)^{2} h_{1}^{2}}+\frac{\left|h_{1}^{k}-h_{1}\right|\left|\left(h_{1}^{k}\right)^{2}+h_{1}^{k} h_{1}+h_{1}^{2}\right|}{\left(h_{1}^{k}\right)^{3} h_{1}^{3}} .
\end{gathered}
$$

We use the Proposition 1 to find two constants $\eta_{1}$ and $\eta_{2}$ such as

$$
\left|\frac{1}{\left(h_{1}^{k}\right)^{2}}-\frac{\alpha}{\left(h_{1}^{k}\right)^{3}}-\left(\frac{1}{h_{1}^{2}}-\frac{\alpha}{h_{1}^{3}}\right)\right| \leq \eta_{1}\left|h_{1}^{k}-h_{1}\right|+\eta_{2}\left|h_{1}^{k}-h_{1}\right| .
$$

So

We have

$$
\left|\frac{1}{\left(h_{1}^{k}\right)^{2}}-\frac{\alpha}{\left(h_{1}^{k}\right)^{3}}-\left(\frac{1}{h_{1}^{2}}-\frac{\alpha}{h_{1}^{3}}\right)\right|^{2} \leq \eta_{3}^{2}\left|h_{1}^{k}-h_{1}\right|^{2} \rightarrow 0 . \quad \text { with } \eta_{3}=2 \max \left(\eta_{1}, \eta_{2}\right) .
$$

$$
\left(\frac{1}{\left(h_{1}^{k}\right)^{2}}-\frac{\alpha}{\left(h_{1}^{k}\right)^{3}}\right)_{k} \quad \text { converges strongly to } \quad \frac{1}{h_{1}^{2}}-\frac{\alpha}{h_{1}^{3}} \quad \text { in } \quad L^{2}\left(0, T ; L^{2}(0,1)\right) .
$$

A similar reasoning ensures the strong convergence of $\left(\frac{1}{\left(h_{1}^{k}\right)^{3}}-\frac{\alpha}{\left(h_{1}^{k}\right)^{4}}\right)_{k}$ to $\frac{1}{h_{1}^{3}}-\frac{\alpha}{h_{1}^{4}}$ in $L^{2}\left(0, T ; L^{2}(0,1)\right)$.

The strong convergence of $\left(\partial_{x} h_{1}^{k}\right)_{k}$ in $L^{2}\left(0, T ; L^{2}(0,1)\right)$ gives us

$$
\left(V\left(h_{1}^{k}\right) \partial_{x} h_{1}^{k}\right)_{k} \quad \text { converges weakly to } \quad V\left(h_{1}\right) \partial_{x} h_{1} \quad \text { in } L^{1}\left(0, T ; L^{1}(0,1)\right) .
$$

\section{Conclusion}

In this paper, we shown the existence of global weak solutions to 1D pollutant transport model. We have considered the existence of molecular interactions between the molecules and we added the term $\varepsilon \partial_{x}^{2} h_{2}$ to the level of the transport equation. This has led us to the use of a molecular interaction force in this work. We obtained a result of existence of 
weak solutions. For our future works, we intend to tend the coefficient $\varepsilon$ (of $\varepsilon \partial_{x}^{2} h_{2}$ ) to 0 . We will also show the existence of strong global solutions of our model.

\section{References}

Bresch, D., \& Desjardins, B. (2003). Existence of global weak solution for 2D viscous shallow water equations and convergence to the quasi-geostrophic model. Comm. Math. Phys., 238(1-3), 211-223. https://doi.org/10.1007/s00220003-0859-8

Bresch, D., Desjardins, B., \& Grard-Varet, D. (2007). On compressible Navier-Stokes equations with density dependent viscosities in bounded domains. J. Math. Pures Appl., 87(9), 227-235. https://doi.org/10.1016/j.matpur.2006.10.010

Bresch, D., Desjardins, B., \& Lin, C. K. (2003). On some compressible fluid models: Korteweg, lubrication and shallow water systems. Communications in partial differential equations, 28(3,4), 843-868. https://doi.org/10.1081/PDE120020499

Fernandez-Nieto, E. D., Narbona-Reina, G., \& Zabsonré, J. D. (2013). Formal derivation of a bilayer model coupling shallow water and Reynolds lubrication equations: evolution of a thin pollutant layer over water. European Journal of Applied Mathematics, 24(6), 803-833. https://doi.org/10.1017/S095679251300020X

Kitavtsev, G., Laurençot, P., \& Niethammer, B. (2011). Weak solutions to lubrication equations in the presence of strong slippage. Methods and Applications of Analysis, 18, 183-202.

Marche, F. (2007). Theoretical and Numerical Study of Shallow Water Models. Applications to Nearshore Hydrodynamics. PhD Thesis, University of Bordeaux.

Mellet, A., \& Vasseur, A., (2007). On the barotropic compressible Navier-Stokes equations. Comm. Partial Differential Equations, 32,(3), 431-452. http://dx.doi.org/10.1080/03605300600857079

Toumbou, B., Le Roux, D., \& Sene, A. (2007) An existence theorem for a 2-D coupled sedimentation shallow-water model. C. R. Math. Acad. Sci. Paris, 344. 443-446. https://doi.org/10.1016/j.jde.2007.12.010

Lions, J. L. (1969). Quelques Mthodes de Rsolution des Problmes aux Limites Non Linaires, Dunod.

Roamba, B., Zabsonré, J. D. D., \& Traoré, S. (2016). Formal derivation and existence of global weak solutions of a two-dimensional bilayer model coupling shallow water and Reynolds lubrication equations. Asymptotic Analysis, 99. 207-239. https://doi.org/10.3233/ASY-16138

Roamba, B., Zabsonré, J. D. D., \& Zongo, Y. (2017). Weak solutions to pollutant transport model in a dimensional case. Submitted in Annals of the University of Craiova, Mathematics and Computer Science Series.

Seemann, R., Herminghaus, S., \& Jacobs, K. (2001). Dewetting Patterns and Molecular Forces: A Reconciliation. PHYSICAL REVIEW LETTERS 24(86), 5534-5537. https://doi.org/10.1103/PhysRevLett.86.5534

Simon, J. (1987). Compact set in the space $L^{p}(0 ; t ; B)$. Ann. Mat. Pura appl. 146(4), 65-96.

Zabsonré J. D. D, Lucas, C., \& Fernndez-Nieto, E. (2009). An energetically consistent viscous sedimentation model. Math. Models Methods Appl. Sci., 19(3), 477-499. https://dx.doi.org/10.1142/S0218202509003504

\section{Copyrights}

Copyright for this article is retained by the author(s), with first publication rights granted to the journal.

This is an open-access article distributed under the terms and conditions of the Creative Commons Attribution license (http://creativecommons.org/licenses/by/4.0/). 